\title{
Familial dysalbuminaemic hyperthyroxinaemia with discordant thyroid function test results: two case reports
}

\author{
Nike KC Lau ${ }^{1 * \dagger}$, MA(Cantab), MBChB (CUHK), Teresa KC Tsui', FHKCPath, FHKAM (Pathology), \\ Jeffrey SS Kwok', FHKCPath, FHKAM (Pathology), Kitty KT Cheung², MRCP, FHKAM (Medicine), \\ CC Chow ${ }^{2}$, FHKCP, FHKAM (Medicine), CK Yeung ${ }^{3}$, FHKCP, FHKAM (Medicine), YP Yuen ${ }^{1 \ddagger}$, FHKCPath, FHKAM (Pathology) \\ ${ }^{1}$ Department of Chemical Pathology, Prince of Wales Hospital, Hong Kong \\ ${ }^{2}$ Department of Medicine and Therapeutics, Prince of Wales Hospital, Hong Kong \\ ${ }^{3}$ Department of Medicine, Tseung Kwan O Hospital, Hong Kong \\ *Corresponding author: lkc416@ha.org.hk \\ † Currently affiliated with Chemical Pathology Laboratory, Department of Pathology, Princess Margaret Hospital, Hong Kong \\ ‡ Currently affiliated with Department of Pathology, Hong Kong Children's Hospital, Kowloon Bay, Hong Kong
}

Hong Kong Med J 2020;26:243-7

https://doi.org/10.12809/hkmj198035

\section{Case report}

Discordant thyroid function test results are a commonly encountered diagnostic challenge. When the inverse log-linear relationship between thyroid stimulating hormone (TSH) and free thyroxine (fT4) is absent, a wide range of differential diagnoses should be considered. This requires the investigatory efforts of endocrinologists and chemical pathologists. We report the diagnostic journey of two Chinese patients with discordant thyroid function test due to familial dysalbuminaemic hyperthyroxinaemia (FDH) [OMIM \#615999].

Patient 1 was a woman with no known family history of thyroid disorder. In 2003, she presented at age 49 years with palpitations and weight loss and was diagnosed with thyrotoxicosis (laboratory test results not available). She was prescribed an antithyroid drug for 18 months and her disease was in apparent remission. She presented again in December 2009, at age 55 years, with weight loss and anxiety. Total thyroxine tested in the private sector was elevated at $186.4 \mathrm{nmol} / \mathrm{L}$ (reference interval $58-155 \mathrm{nmol} / \mathrm{L}$; platform not specified). She was commenced on carbimazole in view of her "relapse" and referred to endocrinology clinic for further management in March 2010. Her first paired TSH and fT4 (Roche Elecsys) results in April 2011 during carbimazole therapy revealed a discordant pattern with a normal TSH of $1.90 \mathrm{mIU} / \mathrm{L}$ and an elevated fT4 at $23.5 \mathrm{pmol} / \mathrm{L}$ (Table 1). Due to the apparent "persistent thyrotoxicosis", she underwent radioactive iodine (RAI) thyroid ablation in May 2011, and carbimazole was stopped after her fT4 was "normalised". Monitoring with fT4 only, her disease appeared to be in remission. Nonetheless her next paired thyroid function test in May 2013 showed elevated TSH $41.8 \mathrm{mIU} / \mathrm{L}$ and low fT4 $11.9 \mathrm{pmol} / \mathrm{L}$, consistent with frank post-RAI hypothyroidism. As a result, she was prescribed thyroxine replacement and her TSH was normalised ( $3.28 \mathrm{mIU} / \mathrm{L})$ by June 2014. She was subsequently followed up in the general outpatient clinic. She was referred again to the endocrine clinic in June 2016 because both TSH and fT4 were elevated despite good compliance with thyroxine treatment. Withholding thyroxine replacement led to elevation of TSH $(19.8 \mathrm{mIU} / \mathrm{L})$ while fT4 $(15.0 \mathrm{pmol} / \mathrm{L})$ and free triiodothyronine $(3.71 \mathrm{pmol} / \mathrm{L})$ were within their respective reference intervals. Chemical pathologists were consulted for discordant thyroid function test results. This specimen was also tested using the Abbott Architect platform and showed similar thyroid function test results. Considering her TSH levels were never suppressed, TSH-dependent thyrotoxicosis was suspected although her serum alpha-subunit level was normal. She was then referred for genetic testing.

Patient 2 was a 34-year-old man who, in February 2004, presented with unintentional weight loss. His thyroid function test revealed normal TSH but elevated fT4 (laboratory test results not available). At follow-up examination, the patient's TSH was re-checked (1.58 mIU/L; Roche Elecsys) but not his fT4, and test results for antithyroglobulin and antimicrosomal antibodies were negative. In view of such normal results, no further followup was arranged until the patient had another thyroid function test on Beckman Coulter Access. This was tested during a preoperative assessment in February 2017 and revealed an elevated fT4 $(36.3 \mathrm{pmol} / \mathrm{L})$ with a non-suppressed TSH $(1.29 \mathrm{mIU} / \mathrm{L})$. He was referred to the endocrinology clinic for further assessment. The patient reported a family history of thyroid disease: his mother had thyrotoxicosis treated with RAI, a maternal uncle had an unspecified thyroid illness, and an elder 
TABLE I. Thyroid function test results (Roche Elecsys unless otherwise specified) of patient I demonstrating the diagnostic difficulty of familial dysalbuminaemic hyperthyroxinaemia compounded by the effects of antithyroid treatment

\begin{tabular}{|c|c|c|c|c|}
\hline Date & Treatment(s) & $\begin{array}{l}\text { TSH (0.27- } \\
4.20 \mathrm{mIU} / \mathrm{L})\end{array}$ & $\begin{array}{c}\text { fT4 (12.0-22.0 } \\
\text { pmol/L) }\end{array}$ & $\begin{array}{c}\text { fT3 (3.10-6.80 } \\
\text { pmol/L) }\end{array}$ \\
\hline 08/03/2010 & \multirow[t]{7}{*}{ Carbimazole } & & 22.7 & \\
\hline $14 / 04 / 2010$ & & 4.5 & & \\
\hline $14 / 06 / 2010$ & & 11.9 & & \\
\hline 04/08/2010 & & 2.7 & & \\
\hline $27 / 10 / 2010$ & & & 21.3 & \\
\hline 19/01/2011 & & & 21.6 & \\
\hline 27/04/2011 & & 1.9 & 23.5 & \\
\hline 15/06/2011 & \multirow{5}{*}{$\begin{array}{l}\text { Carbimazole, } \\
\text { post-RAl }\end{array}$} & 1.3 & 24.1 & \\
\hline 13/07/2011 & & & 19.4 & \\
\hline 03/08/2011 & & 4.6 & 19.4 & \\
\hline $11 / 10 / 2011$ & & & 14.9 & \\
\hline 04/01/2012 & & & 18.3 & \\
\hline 23/05/2012 & \multirow[t]{5}{*}{ Post-RAI } & & 18.2 & \\
\hline $15 / 08 / 2012$ & & & 17.3 & \\
\hline 03/12/2012 & & & 16.4 & \\
\hline $21 / 05 / 2013$ & & 41.80 & 11.9 & \\
\hline 03/10/2013 & & 3.90 & & \\
\hline 07/02/2014 & \multirow{8}{*}{$\begin{array}{l}\text { Post-RAI, } \\
\text { thyroxine } \\
\text { replacement }\end{array}$} & 6.61 & 23.3 & \\
\hline 27/06/2014 & & 3.28 & & \\
\hline $16 / 01 / 2015$ & & 8.04 & 26.8 & \\
\hline 04/02/2015 & & 6.33 & 27.5 & \\
\hline 08/12/2015 & & 7.05 & 24.7 & \\
\hline 22/06/2016 & & 2.97 & 24.5 & \\
\hline 28/09/2016 & & 3.89 & 26.1 & \\
\hline $11 / 01 / 2017$ & & 1.09 & 27.5 & \\
\hline \multirow[t]{2}{*}{$18 / 04 / 2017$} & \multirow[t]{2}{*}{ Post-RAI } & 19.80 & 15.0 & 3.71 \\
\hline & & $11.10^{\star}$ & $11.7^{\star}$ & \\
\hline 05/07/2017 & \multirow{2}{*}{$\begin{array}{l}\text { Post-RAI, } \\
\text { thyroxine } \\
\text { replacement }\end{array}$} & 5.32 & 26.8 & \\
\hline $27 / 12 / 2017$ & & 8.39 & 21.9 & \\
\hline
\end{tabular}

Abbreviations: $\mathrm{fT} 3$ = free triiodothyronine; $\mathrm{fT} 4$ = free thyroxine; $\mathrm{RAl}=$ radioactive iodine; TSH = thyroid stimulating hormone

* Results from Abbott Architect 2-step immunoassay (reference interval:TSH 0.35$4.94 \mathrm{mIU} / \mathrm{L} ; \mathrm{fT} 4$ 9.2-16.9 $\mathrm{pmol} / \mathrm{L})$ brother demonstrated a similar thyroid function test pattern. After discussion with chemical pathologists, subsequent samples were tested on other platforms and showed variable elevation of fT4 (Table 2). Among the four platforms, the degree of elevation in fT4 was greatest on Beckman Coulter Access (209\%$224 \%$ of upper reference limit), followed by Roche Elecsys (155\%). Siemens ADVIA Centaur (101\%), and Abbott Architect (105\%). The results from all four platforms were above the upper reference limit. Free triiodothyronine was also elevated on Beckman Coulter Access (108\%), Abbott Architect (107\%) and Siemens ADVIA Centaur (103\%) platforms, although to a lesser degree. In view of the non-suppressed TSH level, the possibility of a TSH-secreting tumour was considered. Magnetic resonance imaging showed a 2-mm hypo-enhancing anterior pituitary lesion. Biochemical investigations showed normal serum alpha-subunit level and thyrotropin releasing hormone stimulation test. The patient was referred for genetic testing.

Genetic testing for the THRB gene (OMIM "190160) was performed in both patients, in view of possible resistance to thyroid hormone syndrome (OMIM \#188570). No pathogenic variant was detected by polymerase chain reaction and Sanger sequencing. Further testing in both patients for FDH targeting exon 7 of the $A L B$ gene (OMIM "103600; Refseq NG_009291.1/NM_000477.6/NP_000468.1) showed heterozygous c.725G>A p.(Arg242His), a reported pathogenic variant in Chinese. ${ }^{1}$

\section{Discussion}

Familial dysalbuminaemic hyperthyroxinaemia is an autosomal dominant condition caused by variants of albumin, the gene product of $A L B .{ }^{2}$ The prevalence of FDH has been estimated to be $0.01 \%$ in Caucasian populations but much higher (1.0\%-1.8\%) in Hispanic populations. $^{3}$ The prevalence is uncertain in East Asian populations. The variant found in our patients, c.725G >A, has an allele frequency of $0.005437 \%$ (1 in 18392) in East Asian populations, according to the Genome Aggregation Database. However, FDH

TABLE 2. Thyroid function test results of patient 2 on various platforms, without interfering effects of antithyroid treatment

\begin{tabular}{|c|c|c|c|c|c|c|c|c|c|}
\hline Date & Platform & $\begin{array}{c}\text { TSH } \\
\text { (mlU/L) }\end{array}$ & $\begin{array}{c}\text { TSH } \\
\text { reference } \\
\text { interval } \\
\text { (mIU/L) }\end{array}$ & $\begin{array}{c}\text { fT4 } \\
\text { (pmol/L) }\end{array}$ & $\begin{array}{c}\text { fT4 } \\
\text { reference } \\
\text { interval } \\
\text { (pmol/L) }\end{array}$ & $\begin{array}{l}\text { fT4 (as } \\
\text { percentage } \\
\text { of upper } \\
\text { reference } \\
\text { limit) }\end{array}$ & $\begin{array}{c}\text { fT3 } \\
\text { (pmol/L) }\end{array}$ & $\begin{array}{c}\text { fT3 } \\
\text { reference } \\
\text { interval } \\
\text { (pmol/L) }\end{array}$ & $\begin{array}{l}\text { fT3 (as } \\
\text { percentage } \\
\text { of upper } \\
\text { reference } \\
\text { limit) }\end{array}$ \\
\hline $28 / 02 / 2017$ & Beckman Coulter Access & 1.29 & $0.17-4.37$ & 36.3 & $7.7-16.2$ & $224 \%$ & - & - & - \\
\hline $28 / 02 / 2017$ & Roche Elecsys & 1.58 & $0.27-4.20$ & 34.0 & $12.0-22.0$ & $155 \%$ & - & - & - \\
\hline $13 / 03 / 2017$ & Beckman Coulter Access & 2.11 & $0.17-4.37$ & 33.8 & $7.7-16.2$ & $209 \%$ & 6.8 & $2.5-6.3$ & $108 \%$ \\
\hline $13 / 03 / 2017$ & Siemens ADVIA Centaur & 2.15 & $0.25-4.80$ & 23.3 & $12.0-23.0$ & $101 \%$ & 8.2 & $5.1-8.0$ & $103 \%$ \\
\hline $13 / 03 / 2017$ & Abbott Architect & 1.50 & $0.35-4.94$ & 20.0 & $9.0-19.0$ & $105 \%$ & 6.1 & $2.6-5.7$ & $107 \%$ \\
\hline
\end{tabular}

Abbreviations: $\mathrm{fT} 3$ = free triiodothyronine; $\mathrm{fT} 4=$ free thyroxine; $\mathrm{TSH}=$ thyroid stimulating hormone 
is less commonly observed than expected in Hong Kong. The under-recognition of FDH may be due to its asymptomatic nature, and the use of TSH without fT4 for screening of thyroid dysfunction. Our patients were identified only because of their apparent thyrotoxic symptoms and testing of fT4 on an affected platform. Although no treatment is required for $\mathrm{FDH}$, it remains an important entity to recognise due to the potentially devastating complications of inappropriate treatment such as iatrogenic hypothyroidism as in patient 1 , and adverse effects of antithyroid medications such as agranulocytosis. Family cascade screening and counselling should be considered to prevent undesirable misinterpretation and unnecessary treatment of other affected family members. It is strongly discouraged to treat either TSH or fT4 blindly, as is sometimes observed in clinical practice. Once these patients are treated with antithyroid drugs, often with only fT4 used for monitoring, there is an added risk to the development of frank hypothyroidism as the actual fT4 level is lower than the assayed value.

Here we discuss the approach to an elevated fT4 with a non-suppressed TSH in adults, which often requires the concerted efforts of a chemical pathologist and endocrinologist (Table 3). For a more comprehensive review of the general approach to discordant thyroid function test, the reader is advised to consult the excellent review by Koulouri et $\mathrm{al}^{3}{ }^{3}$ Possible differential diagnoses of an elevated fT4 with a non-suppressed TSH in an adult patient include assay interference (including FDH), drugs (such as thyroxine replacement, amiodarone, heparin), non-thyroidal illness (including acute psychiatric disorders), TSH-secreting pituitary adenoma, and resistance to thyroid hormone. ${ }^{3}$

First, clinical correlation of thyroid status with other clinical signs is essential in the interpretation of such thyroid function pattern. It would be sensible to exclude conditions that can be examined by a clinical or drug history, such as the effects of drug therapy, acute psychiatric episodes or non-thyroidal illness. A family history of thyroid disorder should be explored, because it may suggest an inherited condition, such as in patient 2 .

Second, any previous thyroid function results should be reviewed. In patients with thyroid function test results incompatible with clinical features, at least one paired TSH and fT4 and/ or free triiodothyronine should be performed to better detect discordant results. Patients who have previously shown a normal thyroid function pattern are less likely to have inherited conditions such as resistance to thyroid hormone or FDH. For FDH, it is important to note the assay platform used (hinted by the reference intervals provided), as a change of assay from one that is less affected by
TABLE 3. Approach for elevated fT4 with a non-suppressed TSH in an adult patient

Consider differential diagnoses

- Assay interference (eg, biotin, FDH)

- Drugs (eg, thyroxine replacement, amiodarone, heparin)

- Non-thyroidal illness (including acute psychiatric disorders)

- TSH-secreting pituitary adenoma

- Resistance to thyroid hormone

- Disorders of thyroid hormone transport and metabolism (rarely presents in adulthood)

Clinical review

- Assess clinical thyroid status

- Review medical (eg, non-thyroidal illness, acute psychiatric disorders) and drug history

- Explore if any family history of thyroid disease and obtain thyroid function test results, and information of the assay(s) used if possible

Laboratory investigations

- Review of previous thyroid function test results

o Plot TSH and fT4 for the physiological inverse log-linear relationship

o Note the susceptibility to biotin interference and/or FDH of analytical platform(s)

o Has TSH ever been abnormal?

- Investigate possible assay interference

o Repeat test on alternative analytical platform(s)

o Serial dilution (not for free hormone tests)

o Antibody blocking agents

o Polyethylene glycol precipitation (not for free hormone tests)

o Equilibrium dialysis for gold-standard free hormone assessment

Workup for other differential diagnoses

- Tissue markers of thyroid hormone hyperfunction (eg, elevated sex hormonebinding globulin, ferritin, pro-collagen-1-N-terminal peptide, decreased total cholesterol)

- TSH-secreting pituitary adenoma (eg, magnetic resonance imaging of pituitary; serum alpha subunit to TSH molar ratio)

- Genetic testing for resistance to thyroid hormone syndrome (THRB gene) and/ or FDH (ALB gene)

Abbreviations: FDH = familial dysalbuminaemic hyperthyroxinaemia; fT4 = free thyroxine; $\mathrm{TSH}=$ thyroid stimulating hormone

FDH to one that is prone to interference in FDH may give the clinician a false sense of an acquired condition. Moreover, the TSH level in patients with FDH should be normal unless due to thyroid-related treatment, or genuine pituitary or thyroid disease. It is often useful to plot the TSH and fT4 values and try to observe the reciprocal relationship between them. In FDH patients, such a relationship should be largely preserved even after antithyroid treatment, despite an upward shift in fT4 (Fig). This suggests the presence of a functional negative feedback system with the hypothalamus-pituitary-thyroid axis and is less likely to be a case of interference. Thyroxine replacement may further complicate the thyroid function test pattern if thyroxine is taken shortly before blood taking, although the usual thyroid function test requested for patients receiving thyroxine replacement is only TSH.

Third, further investigations for possible assay interference should be discussed with chemical pathologists. In most clinical laboratories, thyroid function tests are performed using automated immunoassays that are prone to interference, for 


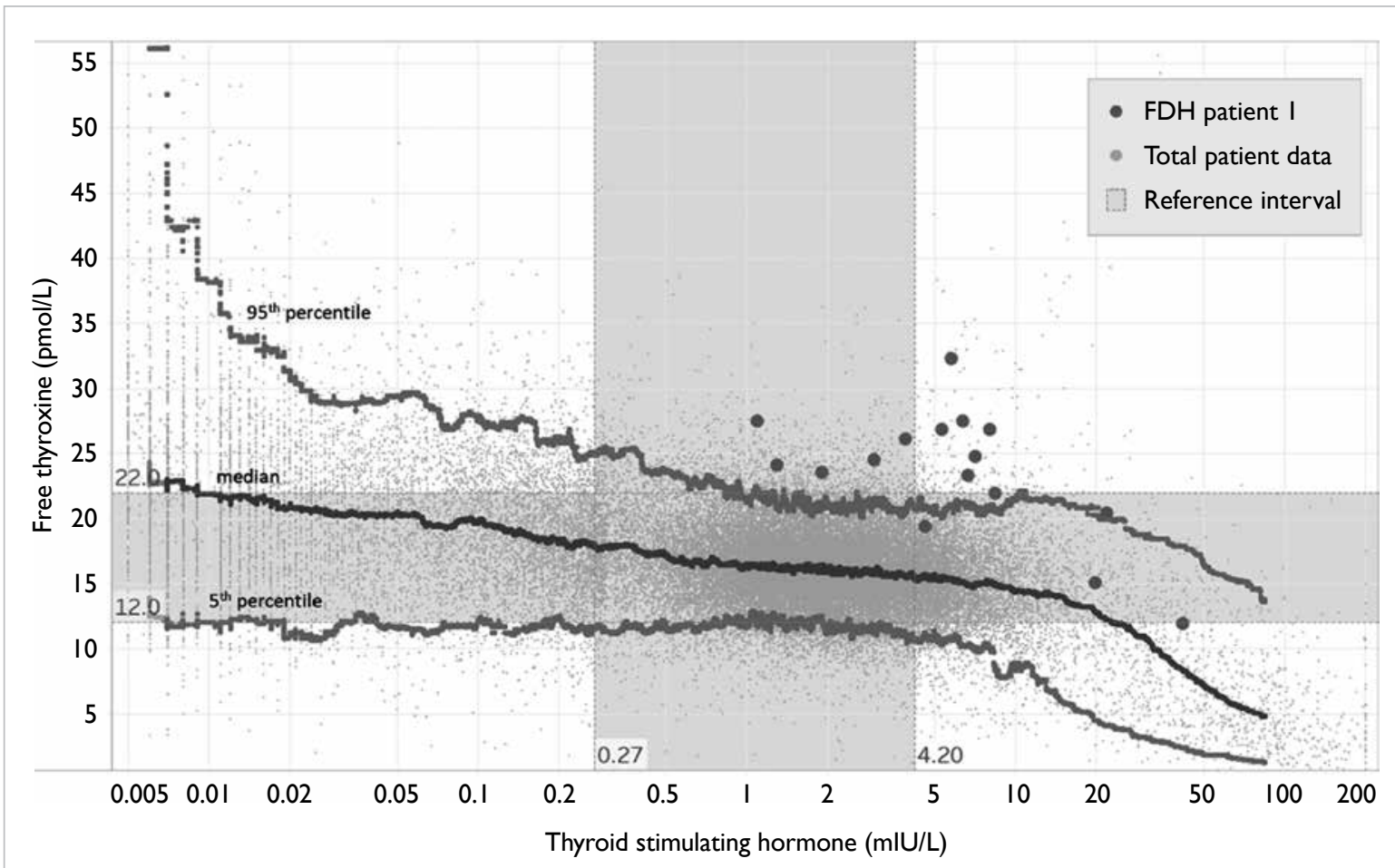

FIG. The 5th, 50th and 95th percentile of free thyroxine plotted against thyroid stimulating hormone using total patient data (in grey dots). The thyroid function test results of patient I (in black dots) demonstrated a largely preserved reciprocal relationship despite an upward shift in free thyroxine

Abbreviation: FDH = familial dysalbuminaemic hyperthyroxinaemia

example by macro-TSH, biotin, anti-streptavidin antibodies, anti-ruthenium antibodies, thyroid hormone autoantibodies or heterophilic antibodies. Further testing such as assay comparison, serial dilution, antibody blocking agents, and polyethylene glycol precipitation may be considered to provide useful clues for possible interference. ${ }^{4}$ Often either the TSH or fT4 assay is affected. Nonetheless, in some cases such as biotin interference, both TSH and fT4 can be affected if they both use the biotinstreptavidin-based separation method in the assay. In patients with FDH, due to an increased binding affinity of thyroxine to albumin, total thyroxine is elevated, and to a lesser extent total triiodothyronine, although fT4 measured using equilibrium dialysis, which only minimally disrupts the equilibrium between the free and the bound portion of thyroxine, should be normal. ${ }^{4}$ Nevertheless, the presence of this albumin variant has been shown to lead to overestimation of fT4 levels in Roche Elecsys and Siemens Immulite (competitive one-step assays), whereas Abbott Architect (two-step assay) is less affected, because the thyroxine analogue does not come into contact with the variant albumin. ${ }^{5}$ Beckman Coulter Access, a two-step assay, is an exception as it also shows significant overestimation of fT4. The practical implication is that when FDH is suspected, one should examine the type of fT4 assay used, and repeat testing on a less affected platform.

Apart from the usual investigations for TSHsecreting pituitary adenoma such as magnetic resonance imaging of the pituitary and serum alpha subunit to TSH molar ratio, or genetic testing for resistance to thyroid hormone, further biochemical testing of the thyroid status of the patient should be considered. Tissue markers of thyroid hormone hyperfunction such as increased sex hormonebinding globulin, ferritin, pro-collagen-1-Nterminal peptide, and decreased cholesterol may provide additional evidence of a thyrotoxic state. ${ }^{6}$ The absence of these may suggest resistance to thyroid hormone, or assay interference. Further investigations such as serum thyroglobulin, thyroid antibodies (including anti-TSH receptor antibodies), thyroid scan, and ultrasonography may provide additional clues to the thyroid status in difficult cases. However, their use should be discussed with endocrinologists or relevant specialists to ensure optimal test utilisation and interpretation.

In summary, although both our patients ultimately received the correct diagnosis of FDH after collaboration between endocrinologists and chemical pathologists, the complications associated with misinterpretation and inappropriate treatment 
illustrate the importance of a good understanding of the analytical pitfalls of thyroid function test assays and proper follow-up investigations and management of discordant thyroid function test. Clinicians should be aware of the approach to discordant thyroid function, especially those who treat thyroid conditions.

\section{Author contributions}

Concept or design: All authors.

Acquisition of data: All authors.

Analysis or interpretation of data: NKC Lau, TKC Tsui, JSS Kwok.

Drafting of the article: NKC Lau, TKC Tsui, JSS Kwok.

Critical revision for important intellectual content: All authors.

All authors had full access to the data, contributed to the study, approved the final version for publication, and take responsibility for its accuracy and integrity.

\section{Conflicts of interest}

All authors have disclosed no conflicts of interest.

\section{Acknowledgement}

We would like to thank all laboratory and clinical staff who assisted in the cross-platform testing and management of the patients.

\section{Funding/support}

This case report received no specific grant from any funding agency in the public, commercial, or not-for-profit sectors.

\section{Ethics approval}

Informed consent was obtained from all patients.

\section{References}

1. Tiu SC, Choi KL, Shek CC, Lau TC. A Chinese family with familial dysalbuminaemic hyperthyroxinaemia. Hong Kong Med J 2003;9:464-7.

2. Kragh-HansenU,GallianoM,MinchiottiL.Clinical, genetic, and protein structural aspects of familial dysalbuminemic hyperthyroxinemia and hypertriiodothyroninemia. Front Endocrinol (Lausanne) 2017;8:297.

3. Koulouri O, Moran C, Halsall D, Chatterjee K, Gurnell M. Pitfalls in the measurement and interpretation of thyroid function tests. Best Pract Res Clin Endocrinol Metab 2013;27:745-62.

4. Favresse J, Burlacu MC, Maiter D, Gruson D. Interferences with thyroid function immunoassays: clinical implications and detection algorithm. Endocr Rev 2018;39:830-50.

5. Cartwright D, O'Shea P, Rajanayagam O, et al. Familial dysalbuminemic hyperthyroxinemia: a persistent diagnostic challenge. Clin Chem 2009;55:1044-6.

6. Singh BK, Yen PM. A clinician's guide to understanding resistance to thyroid hormone due to receptor mutations in the TR $\alpha$ and TR $\beta$ isoforms. Clin Diabetes Endocrinol 2017;3:8. 